\title{
Fatores genéticos e ambientais sobre a probabilidade de prenhez precoce em bovinos Caracu
}

\author{
Genetic and environment factors on probability of early pregnancy of Caracu cattle
}

\author{
Maira Mattar ${ }^{\mathrm{I}}$ Sarah Laguna Meirelles ${ }^{\mathrm{I}}$ João Ademir de Oliveira ${ }^{\mathrm{II}}$ \\ Ana Carolina Espasandin ${ }^{\mathrm{III}}$ Sandra Aidar de Queiroz ${ }^{\mathrm{IV}}$
}

RESUMO

Em bovinos, critérios de seleção, associados à precocidade sexual, vêm sendo atualmente pesquisados para a sua inclusão em programas de seleção, visando à diminuição da idade de entrada dos animais em reprodução, com conseqüências positivas sobre a receita do rebanho. $O$ objetivo deste trabalho foi estimar a herdabilidade da probabilidade de prenhez precoce (PPP) de fêmeas Caracu, característica associada à puberdade e que pode ser considerada como indicadora do início da atividade reprodutiva, usando para isso, um arquivo com 5.273 observações. A significância dos efeitos da produção de leite da mãe na primeira lactação, da idade da mãe ao parto e da idade à desmama das novilhas sobre a PPP, foi analisada por meio do procedimento logístico de regressão. Empregou-se a análise Bayesiana para a estimação de herdabilidade da PPP mediante um modelo de touro, onde foram incluídos os efeitos de grupo de contemporâneos, produção de leite na primeira lactação, touro e resíduo. A média a posteriori da herdabilidade da PPP foi de 0,13, que, embora baixa, evidenciou a presença de ação gênica aditiva na determinação desta característica, o que possibilitaria a obtenção de progresso genético na mesma.

Palavras-chave: bovino Caracu, estatística Bayesiana, probabilidade de prenhez precoce.

\section{ABSTRACT}

Selection criteria associated to sexual precocity have increased their importance and in order to reduce the age at first calving they have been including in selection programs with positive consequences to the profit of the herds. The present study estimated the heritability for probability of early pregnancy (PPP), a trait associated with puberty, that could be considered as indicative of the beginning of the reproductive activity in females, using records on 5273 females. In order to verify the significance of the effects of milk yield in first lactation, age at calving of the dam and weaning age of the heifer on PPP logistic regression was applied. Heritability for PPP was estimated by Bayesian method, using a sire model. This model included the effects of contemporary group, milk yield in first lactation (in classes), sire and residual. The "posteriori" mean of heritability was 0.13. This value, although not high, showed the presence of genetic additive action on the determination of this trait.

Key words: Bayesian statistic, Caracu cattle, probability of precocious pregnancy.

\section{INTRODUÇÃO}

A prenhez de novilhas é uma característica que vem, atualmente, merecendo interesse dos pesquisadores e foi definida por ELER et al. (2004) como sendo a constatação de que uma novilha concebeu e permaneceu prenhe até o diagnóstico de gestação, uma vez ter tido oportunidade de ser acasalada. Esta característica é medida diretamente na novilha, levando em conta a sua fertilidade inerente; por isso, é uma característica econômica de interesse.

IPrograma de Pós-graduação em Zootecnia, Faculdade de Ciências Agrárias e Veterinária (FCAV), Universidade Estadual de São Paulo (Unesp), Jaboticabal, SP, Brasil.

IDepartamento de Ciências Exatas, FCAV, Unesp, Jaboticabal, SP, Brasil.

IIIDepartamento de Producción Animal y Pasturas, Universidad De La República (UDELAR), Paysandú, Uruguay.

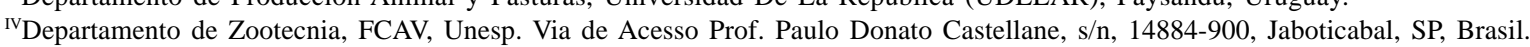

E-mail: saquei@fcav.unesp.br. Autor para correspondência. 
A medida atual de precocidade sexual em fêmeas é a probabilidade de prenhez aos 14 meses, a qual tem expressão fenotípica discreta, com resposta de sucesso (1) ou fracasso (0). Entretanto, para a obtenção desta característica, faz-se necessário submeter novilhas à monta em idades variando de 12 a 16 meses, manejo ainda pouco comum mesmo entre criadores envolvidos em programas de melhoramento genético de bovinos.

Assim, visando a contornar a resistência de parte dos criadores de bovinos em aplicar este manejo às fêmeas e também a possibilitar a inclusão de um maior número de animais nas avaliações genéticas, ATENCIO (2000) sugeriu uma nova abordagem para o estudo da precocidade sexual de fêmeas, mediante a utilização de uma característica discreta, denominada por ele de probabilidade de prenhez precoce. Por esta abordagem, todas as fêmeas nascidas em um rebanho de bovinos de corte, a princípio, seriam destinadas à reposição. Se isso não fosse verificado, a razão seria a ocorrência de seleção prévia (natural ou não) baseada, intuitivamente, em critérios reprodutivos. Desta maneira, todas as fêmeas desmamadas passariam a fazer parte da amostra considerada. Aquelas que entrassem efetivamente para o rebanho receberiam escore 1 e as que fossem descartadas receberiam escore 0 .

Para a análise de características categóricas como a PPP, os procedimentos com modelos lineares (ML) não são apropriados para quantificar a natureza discreta destas (SILVA et al., 2003), pois não consideram a distribuição não-normal do erro associado com a característica observada em categorias. A partir desta realidade, procedimentos empregando modelos nãolineares foram elaborados combinando-se as teorias de modelos lineares e de modelos de limiar. Características de limiar (threshold) foram definidas por FALCONER \& MACKAY (1996) como aquelas que variam descontinuamente, mas não apresentam herança mendeliana simples. O limiar ou threshold determina o nível de propensão no qual o fenótipo apresenta sucesso ou fracasso na expressão da característica.

Com os avanços na capacidade de processamento e armazenamento de informações pelos computadores, os métodos Bayesianos vêm sendo utilizados para a estimação dos componentes de variância de dados categóricos, como aqueles que utilizam resultados da teoria de cadeias de Markov, destacando-se, dentre eles, a amostragem de Gibbs. A metodologia Bayesiana diferencia-se da clássica (frequentista), pois os parâmetros são vistos como variáveis aleatórias, cujo comportamento é regulado por uma distribuição de probabilidade, que proporciona informação inicial ou a priori dos parâmetros, antes mesmo de se obter os dados. Essa informação a priori é então combinada com a informação fornecida pelos dados, produzindo-se um valor para o parâmetro que representa a nova opinião sobre o mesmo. A combinação da informação a priori com a informação proveniente dos dados resulta na chamada distribuição a posteriori do parâmetro, que é utilizada para o cálculo de sua estimativa. Além disso, no modelo de avaliação, todos os efeitos são considerados aleatórios e com as mesmas propriedades descritas anteriormente. O objetivo deste estudo foi estimar a herdabilidade da característica probabilidade de prenhez precoce (PPP) por meio da análise Bayesiana, utilizando dados de rebanhos da raça Caracu.

\section{MATERIAL E MÉTODOS}

Para o estudo da probabilidade de prenhez precoce (PPP), foram analisados dados de fêmeas da raça Caracu, nascidas no período de 1970 a 1998, de dois rebanhos localizados nos municípios de Poços de Caldas e Bambuí, respectivamente, em Minas Gerais. Até o início de 1980, a seleção dos animais foi baseada na produção de leite das mães. A partir de 1994, todas as novilhas passaram a ter a oportunidade de entrar em reprodução, sendo descartadas as não-prenhes e as vacas com baixa produção de leite à primeira lactação. Os animais foram criados em regime pastoril, recebendo, no inverno, silagem de milho (Zea mays, $L$ ). Todas as vacas em lactação receberam $2,0 \mathrm{~kg}$ de ração concentrada no cocho, independentemente do nível de produção de leite. Os bezerros em amamentação receberam 1,0kg de ração concentrada, de 60 dias após o nascimento até a desmama, que ocorreu por volta dos 300 dias de idade. As novilhas foram colocadas em reprodução com peso próximo aos $350 \mathrm{~kg}$, com cerca de 18 meses de idade. O manejo reprodutivo foi por monta natural, utilizando um touro para cada retiro e para cada lote de, em média, 50 fêmeas. As montas ocorreram de abril a fevereiro, evitando-se, deste modo, nascimento em dezembro e janeiro, que são meses com maior incidência de chuvas

A variável grupo de contemporâneos (GC) foi formada por animais pertencentes ao mesmo rebanho, nascidos no mesmo ano e estação. A estação de nascimento foi definida pelas classes: $1=$ setembro a novembro; 2 = dezembro, janeiro e fevereiro; 3 = março a maio e 4 = junho a agosto. A variável discreta probabilidade de prenhez precoce (PPP) foi definida pelo rastreamento na genealogia dos dados de identificação das fêmeas nascidas nos rebanhos. As fêmeas (novilhas) identificadas como reprodutoras no arquivo de genealogia receberam o valor 1 e as que 
não foram incorporadas ao rebanho, portanto ausentes no arquivo de reprodutores, por motivos de nãoprenhez ou outros motivos não controlados, receberam o valor zero.

Os efeitos da produção de leite da mãe na primeira lactação (PL), da idade da mãe ao parto (IVP) e da idade da novilha à desmama (ID) sobre a PPP foram estudados organizando-se estas variáveis em intervalos de classes. A PL foi assim classificada: classe $1=\mathrm{PL}<1200 \mathrm{~kg} /$ lactação, classe $2=1200 \mathrm{~kg}<\mathrm{PL}<$ $2000 \mathrm{~kg} /$ lactação e classe 3 = PL > 2000kg / lactação. Para a IVP, 3 classes, em meses, foram definidas, a saber: $1=23 \leq \operatorname{IVP}<33,2=33 \leq \operatorname{IVP}<43$ e $3=\operatorname{IVP} \geq 43$; enquanto que a ID, em dias, também foi fracionada em: classe $1=100 \leq$ ID $<250$, classe $2=250 \leq$ ID $<300$ e classe $3=\mathrm{ID} \geq 300$ dias.

Após a edição dos dados, o arquivo apresentou 5.273 observações de fêmeas com informações de pai, mãe, GC, classes de PL, IVP e ID e PPP.

Com o intuito de verificar a significância dos efeitos de PL, IVP e ID sobre a PPP, os dados foram analisados por procedimento que ajusta modelos de regressão linear logístico para dados de resposta binária ou ordinal, pelo método da máxima verossimilhança.

Para a obtenção das distribuições $a$ posteriori dos componentes de variância e estimativa da herdabilidade de PPP foi utilizado o software MTGSAM_threshold(VAN TASSEL \& VAN VLECK, 1995), por meio de modelo de touro que incluiu os efeitos genéticos de touro, residual, além dos efeitos significativos na análise anterior, isto é, PL e GC.

O modelo de limiar usado relaciona a resposta observada na escala categórica a uma escala subjacente normal contínua. Assumindo-se que a escala subjacente tem distribuição normal: $\mathrm{U} \mid$ è N (Wè, I $\sigma \mathrm{e}^{2}$ ); em que: U é o vetor da escala base de ordem r; è' = (EF', a') é o vetor dos parâmetros de locação de ordem s com EF (definidos sob o ponto de vista frequentista, como efeito fixos) e "a" (como efeito aleatório); W é uma matriz de incidência conhecida de ordem r por s; I é uma matriz de identidade de ordem r por $r$; e $\sigma_{\mathrm{e}}^{2}$ é a variância residual.

Assumiu-se, para os efeitos genéticos e os resíduos, distribuições iniciais normais multivariadas: $\mathrm{P}\left(\mathrm{a} \mid \sigma_{\mathrm{a}}{ }^{2}\right) \sim \mathrm{N}\left(0, \sigma_{\mathrm{a}}^{2}\right) ; \mathrm{P}\left(\mathrm{e} \mid\left(\sigma_{\mathrm{e}}^{2}\right) \sim \mathrm{N}\left(0, \mathrm{I} \sigma_{\mathrm{e}}^{2}\right)\right.$

Como a variância residual $\left(\sigma_{\mathrm{e}}^{2}\right)$ não é estimável em modelos de limiar, GIANOLA \& FOULLEY (1983) sugeriram atribuir o valor 1 para a mesma $\left(\sigma_{\mathrm{e}}{ }^{2}=1\right)$.

Foram definidas distribuições prioris nãoinformativas para todos os efeitos e para as variâncias genéticas. Espera-se, com esse tipo de distribuição, que a informação a priori tenda a ter pouca influência nas estimativas.

Após a definição dos parâmetros do modelo, pode ser estabelecido que o encadeamento das duas escalas, categórica e contínua, é proporcional a:

$$
\mathrm{P}(\mathrm{y} v=0 \mid \mathrm{t}, \mathrm{è})=\mathrm{P}(\mathrm{Uv}<\mathrm{è} \mid \mathrm{t}, \mathrm{è})=\ddot{\mathrm{O}}((\mathrm{t}-\mathrm{w} \text {, }
$$

$v$ è) / бe);

em que yv é a variável resposta para a $\mathrm{v}^{\text {th }}$ observação; t é o valor do limiar que, por não ser estimável, será fixado um valor arbitrário; Uv é o valor da variável subjacente para a mencionada observação; Ö ( ) é a função de distribuição cumulativa de uma variável normal padrão; e w’v é um vetor coluna de incidência que une è à observação $\mathrm{v}^{\text {th }}$. Por serem as observações condicionalmente independentes, dado è, a função de verossimilhança é definida pelo produto das contribuições de cada registro.

De acordo com a inferência Bayesiana, o produto da distribuição anterior pela função de verossimilhança é proporcional à distribuição posterior conjunta de è e $\sigma_{a}^{2}$. Foram então obtidas as distribuições posteriores marginais dos parâmetros usando a amostragem de Gibbs (GELFAND \& SMITH, 1990).

O modelo de touro utilizado incluiu os efeitos de grupo de contemporâneos (GC), classe de produção de leite (PL), touro e resíduo. O arquivo para a análise continha 5.273 informações, 111 GC e 435 touros. Os GC que apresentaram apenas uma classe de variávelresposta, sucesso ou fracasso, foram excluídos da análise, de maneira a evitar sub ou superestimação (BERGER, 1994). O modelo da análise pode ser descrito como: $\mathrm{Y}=\mathrm{X} \beta+\mathrm{Wa}+\mathrm{e}$; em que: $\mathrm{Y}=$ vetor de observações; $\beta$ = vetor de efeitos fixos; $a=$ vetor de efeito do touro; e = vetor de efeitos residuais; sendo $\mathrm{X}$ e W matrizes de incidência associadas aos efeitos apropriados a Y.

O comprimento das cadeias de Markov utilizado no cálculo da estimativa de herdabilidade de PPP foi de 500.000 ciclos, com período de descarte amostral dos 10.000 vetores iniciais, para permitir que a distribuição inicial, fornecida como priori, não interferisse nos resultados (SANTOS et al., 2004). Para evitar a redundância das informações causada pelas correlações seriais entre vetores gerados subseqüentemente, foram tomados vetores a cada 380 ciclos, para se obter a amostra utilizada na análise. Esse valor foi obtido por análise prévia dos valores de variância genética aditiva utilizando o programa Gibanal, sob sistema operacional DOS, desenvolvido por VAN KAAM (1997). 
Para cada vetor daquela amostra, foi calculada a herdabilidade, segundo a fórmula: $h^{2}=4$ $\sigma^{2}{ }_{s} /\left(1+\sigma_{s}^{2}\right)$; em que: $\sigma_{s}^{2}=$ variância de touro.

Após a obtenção dos valores das herdabilidades para todos os vetores da amostra, obteve-se a média de todos os valores gerados, que é a estimativa Bayesiana desse parâmetro.

\section{RESULTADOS E DISCUSSÃO}

Na análise pelo procedimento logístico dos efeitos da produção de leite da mãe na primeira lactação (PL), de idade da novilha à desmama (ID) e da idade da mãe ao parto (IVP), sobre a PPP de fêmeas Caracu, observou-se que apenas PL teve influência significativa. Este resultado, provavelmente, ocorreu em razão de o criador ter selecionado os animais por um longo período de tempo, baseando-se na produção de leite de suas mães. Por outro lado, as variáveis ID e IVP não apresentaram efeitos significativos sobre a PPP, resultado que também pode ser justificado pelo manejo praticado nas propriedades, que até 1994 não desafiava as novilhas à reprodução, ou seja, estas eram expostas aos touros com cerca de dois anos de idade. Desta maneira, como as novilhas eram desmamadas em torno de 300 dias, e a inclusão destas ao rebanho era feita em idade bem posterior à desmama, e os efeitos ID e IVP já apresentavam-se diluídos por essa ocasião.

A princípio, a análise Bayesiana para a obtenção das variâncias genéticas foi realizada usandose um modelo animal, incluindo-se os efeitos genético direto do animal, genético materno, de ambiente permanente da mãe da novilha, PL e GC. Entretanto, a utilização deste modelo, provocou o fenômeno blowing-up (HOESCHELE et al., 1995), ou seja, as iterações eram encerradas antes de atingirem o critério de convergência estipulado e as estimativas de variância genética aditiva das amostras apresentavamse sempre crescentes. Esta dificuldade tem sido descrita na utilização de modelos de limiar quando os efeitos fixos apresentam muitos níveis (HOESCHELE \& TIER, 1995; MORENO et al., 1997), como neste estudo com 111 GC e/ou quando existe grande variabilidade entre os efeitos. Segundo BERGER (1994), em razão destas dificuldades, a maior parte dos estudos com aplicação de modelos não-lineares tem utilizado modelo de touro em vez de modelo animal. Deste modo, foi utilizado modelo de touro que usa as informações das filhas (meias irmãs e irmãs germanas), reduzindo o tamanho da matriz de parentesco, concentrando as observações das características em um menor número de animais, o que possibilitou a convergência da análise, mediante o emprego de um modelo de limiar.

Na população Caracu aqui analisada, a média de animais com resposta 1 (sucesso) para PPP foi $46 \%$. Isto significa que, para esta população, um touro médio, isto é, com diferença esperada na progênie igual a zero, apresentaria filhas com probabilidade de prenhez precoce igual a $46 \%$. No entanto, como a escala de porcentagem é inapropriada para expressar a incidência (valor do fenótipo medido em proporção), nas análises genéticas, as incidências devem ser convertidas em “susceptibilidade média” normalmente distribuída; calculada por meio do desvio normal (x), que é o desvio do limiar em relação à média. A susceptibilidade média da característica PPP foi - 0,10 $\sigma_{\mathrm{p}}$ para os animais que apresentaram o fenótipo de sucesso, em que $\sigma_{p}$ é o desvio padrão fenotípico da PPP, e se apresenta negativo por situar-se à esquerda da média.

A tabela 1 apresenta as médias (x); medianas (Mi) e modas (Mo) a posteriori dos componentes de variância e da herdabilidade da PPP. Observa-se que todas as medidas de posição a posteriori da herdabilidade de PPP foram de baixa magnitude, ou seja, iguais a 0,13. Devido a distribuição a posteriori das estimativas obtidas neste estudo possuir densidade simétrica (Figura 1), os valores de x e Mi resumem a medida da distribuição. No entanto, segundo WRIGHT et al. (2000), a moda é a mais aconselhável medida de posição das distribuições $a$ posteriori, pois aponta o valor de maior freqüência. $\mathrm{O}$ intervalo de credibilidade para o referido parâmetro, adotando-se probabilidade de $90 \%$, variou de 0,06 a 0,21 .

O valor da herdabilidade de uma característica é importante para direcionar o programa

Tabela 1 - Tamanho da amostra (N), Média (x), Mediana (Mi), Moda (Mo) e Intervalo de Credibilidade (IC) dos componentes de variância de touro $\left(\sigma^{2}\right)$ e de herdabilidade $\left(\mathrm{h}^{2}\right)$ da probabilidade de prenhez precoce de fêmeas Caracu.

\begin{tabular}{|c|c|c|c|c|c|c|c|}
\hline Estimativas & $\mathrm{N}$ & $\mathrm{x}$ & Mi & Mo & IC & Mínimo & Máximo \\
\hline$\sigma_{s}^{2}$ & 1290 & 0,03 & 0,03 & 0,03 & {$[0,01 ; 0,05]$} & 0,00 & 0,08 \\
\hline$\sigma^{2}{ }^{1}$ & 1290 & 1,00 & 1,00 & 1,00 & -------- & 1,00 & 1,00 \\
\hline$h^{2}$ & 1290 & 0,13 & 0,13 & 0,13 & {$[0,06 ; 0,21]$} & 0,00 & 0,31 \\
\hline
\end{tabular}

${ }^{1} \sigma^{2}{ }_{\mathrm{e}}=$ Estimativa da variância residual que foi assumida constante e igual a 1. 


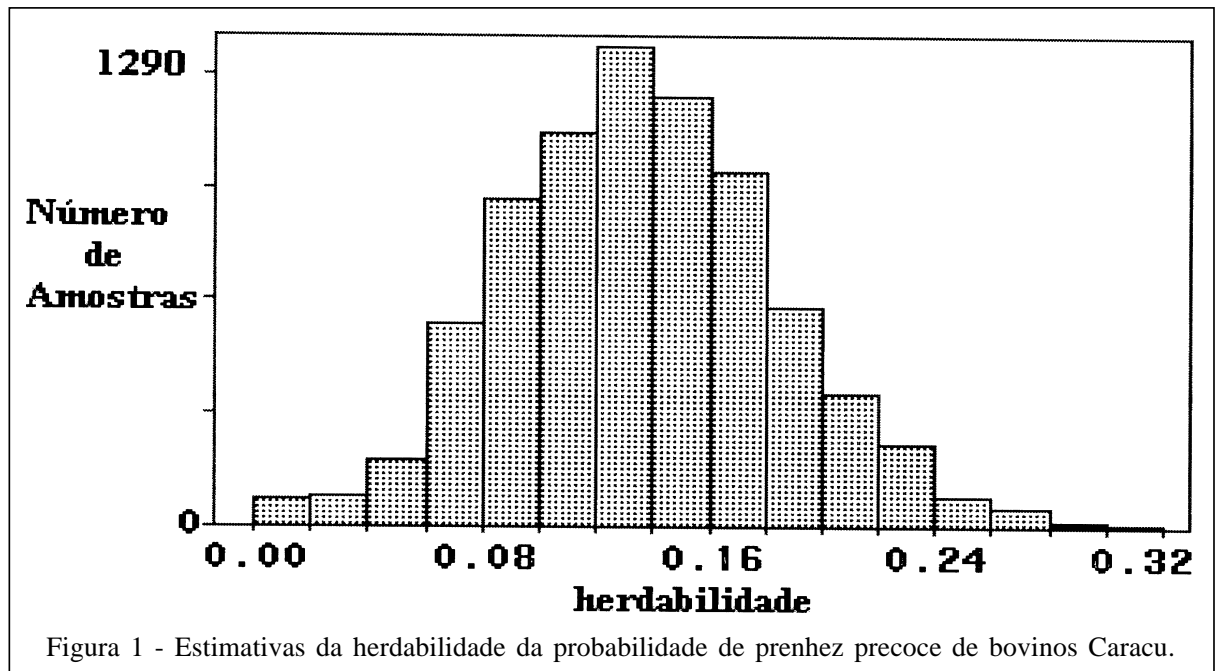

de seleção. Deste modo, comparando-se este resultado com estimativas de herdabilidade de outras características reprodutivas, utilizadas como indicadoras de precocidade sexual, como a idade ao primeiro parto, que, para a raça Caracu, tal como relatado por QUEIROZ (2002), foi igual a 0,09, o uso da PPP como critério de seleção seria uma alternativa viável, uma vez que poderia conferir maior resposta à seleção, em termos de precocidade sexual. Além disso, a PPP por ser facilmente obtida e não requerer mudanças de manejo, sendo é uma característica que pode ser usada por todas os criadores que façam controle zootécnico do rebanho.

Os resultados obtidos neste estudo confirmam os relatados por outros autores que estimaram herdabilidade para prenhez de novilhas em raças de origem européia (Bos taurus) como a raça Caracu. DOYLE et al. (1996), estudando a PP14 de vacas Angus e EVANS et al. (1999), com fêmeas da raça Hereford, estimaram, respectivamente, os valores de 0,21 e 0,13.

A pequena magnitude da estimativa de herdabilidade da PPP obtida neste estudo pode ser reflexo do próprio processo de formação da raça Caracu. Esta se originou do acasalamento ao acaso entre bovinos de diversas raças européias trazidos pelos colonizadores portugueses ao Brasil, ao longo de muitos anos (CARVALHO DIAS, 1948). Acasalamentos ao acaso entre animais de raças européias da Península Ibérica, aliados à intensa seleção natural, favoreceram animais com melhores desempenhos reprodutivos e mais adaptados às condições tropicais. Este processo, possivelmente, resultou em bovinos mais precoces sexualmente, portanto, com menor variabilidade genética para idade à puberdade. Os valores relatados para as estimativas de herdabilidade da probabilidade de prenhez aos 14 meses de bovinos da raça Nelore foram de magnitudes maiores 0,49 (SILVA et al., 2002) do que os obtidos para as raças de origem européias, como a Caracu, reflexo da maior variabilidade genética dos rebanhos ainda pouco selecionados para precocidade sexual. Cabe ressaltar, no entanto, que as variações nas estimativas de herdabilidade entre os diferentes estudos podem ser atribuídas não só à composição genética do rebanho, mas também ao manejo dado em cada propriedade, ao tamanho da população e aos procedimentos analíticos adotados (SNELLING et al., 1995). Assim, nesta pesquisa, a metodologia Bayesiana empregada no estudo da característica PPP, além de possibilitar a estimação da herdabilidade de uma característica discreta e com apenas duas classes, permitiu também calcular intervalos de credibilidade estreitos para o parâmetro, resultando em boa precisão da estimativa.

\section{CONCLUSÃO}

O uso da metodologia Bayesiana possibilitou estimar a herdabilidade da característica binária probabilidade de prenhez precoce. A seleção baseada neste critério reprodutivo poderá trazer progressos moderados na precocidade sexual de fêmeas da raça Caracu.

\section{AGRADECIMENTOS E APRESENTAÇÃO}

Os autores agradecem ao Zootecnista M.Sc. André Stein Carvalho Dias, pela cessão dos dados usados nesta pesquisa; ao Professor Adhemar Sanches, pelo auxílio na compreensão da metodologia Bayesiana e ao Conselho Nacional de Desenvolvimento Científico e Tecnológico (CNPq), pelo apoio financeiro concedido. 
Este trabalho é parte da dissertação de mestrado da primeira autora, apresentada ao programa de Pós-graduação em Zootecnia, área de concentração em Genética e Melhoramento Animal, Faculdade de Ciências Agrárias e Veterinárias, Campus de Jaboticabal - UNESP.

\section{REFERÊNCIAS}

ATENCIO, A. Predicciones genéticas de la fertilidad en la hembra cebú. In: CONGRESO INTERNACIONAL DE TRANSFERENCIA TECNOLÓGICA AGROPECUÁRIA: Cría y Mejoramiento Genético, 8., 2000, Mariano R. Alonso. Anales... Asunción: CEA, 2000. 117p. p.29-40.

BERGER, P.J. Genetic prediction for calving ease in the United States: data models, and use by the dairy industry. Journal of Dairy Science, v.77, n.4, p.1146-1153, 1994.

CARVALHO DIAS, E. O gado Caracu sob o sistema de retiros. Revista Ceres, Viçosa, v.7, n.22, p.338-402, 1948.

DOYLE, S.P. et al. Genetic parameter estimates for heifer pregnancy rate and subsequent breeding rate in Angus cattle. Journal of Animal Science, v.74, n.1, p.117, 1996.

ELER, J.P. et al. Additive genetic relationships between heifer pregnancy and scrotal circumference in Nelore cattle. Journal of Animal Science, v.82, n.9, p.2519-2527, 2004.

EVANS, J.L. et al. Aditive genetic relationship between heifer pregnancy and scrotal circumference in Hereford cattle. Journal of Animal Science, v.77, n.10, p.2621-2628, 1999.

FALCONER, D.S.; MACKAY, T.F.C. Introduction to quantitative genetics. 4. And Tech., Harlow, UK: Longman Science,1996. 464p.

GELFAND, A.E.; SMITH, A.F.M. Sampling based approaches to calculating marginal densities. Journal of the American Statistical Association, v.85, p.398-409, 1990.

GIANOLA, D.; FOULLEY, J.L. Sire evaluation for ordered categorical data with a threshold model. Genetics Selection Evolution, v.15, p.201-224, 1983.

HOESCHELE, I., et al. Multiple traits genetic evaluation for one polycothomous trait and several continuous traits with missing data and unequal models. Journal of Animal Science, v.73, n.6, p.1609-1627, 1995.
HOESCHELE, I..; TIER, B. Estimation of variance components of threshold characters by marginal posterior modes and means via Gibbs sampling. Genetics Selection Evolution, v.27, n.6, p.519-540, 1995.

MORENO, C. et al. On biased inference about variance components in the binary threshold model. Genetics Selection Evolution, v.29, n.2, p.145-160, 1997.

QUEIROZ, S.A. Valores econômicos e índices de seleção para características biológicas dos objetivos de seleção de um núcleo de bovinos da raça Caracu. 2002. $194 \mathrm{f}$. Tese (Livre-docência) - Faculdade de Ciências Agrárias e Veterinárias - Jaboticabal, Universidade Estadual Paulista.

SANTOS, G.B. et. al. Inferência bayesiana na estimação de componentes de variância de bovinos Simental. In: SIMPÓSIO DA SOCIEDADE BRASILEIRA DE MELHORAMENTO ANIMAL, 5., 2004, Pirassununga, SP. Anais... Pirassununga: Sociedade Brasileira de Melhoramento Animal, 2004. CDRoom.

SILVA, J.A.II V. et al. Estimação de parâmetros genéticos para probabilidade de prenhez aos 14 meses e altura na garupa em bovinos da raça Nelore. Revista Brasileira de Zootecnia, v.32, n.5, p.1141-1146, 2003.

SILVA, J.A.II.V. et al. Estimação da herdabilidade da probabilidade de prenhez aos 14 meses usando inferência bayesiana. In: REUNIÃO ANUAL DA SOCIEDADE BRASILEIRA DE ZOOTECNIA, 39., 2002, Recife, PE. Anais... Recife: Sociedade Brasileira de Zootecnia, 2002. CD-Rom.

SNELLING, W.M. et al. Application of continuous and binary trait methods to reproductive measure of Hereford cattle. Journal of Animal Science, v.74, suppl.1, p.115, 1995.

VAN KAAM, J.B.C.H.M. GIBANAL - Analyzing program for Markov Chain Monte Carlo sequences. Version 2.4. Netherlands: Department of Animal Breeding. Wageningen Agricultural University, 1997. CD-rom

VAN TASSEL, C.P., VAN VLECK, L.D. A manual for use of MTGSAM. A set of fortran programs to apply gibbs sampling to animal models for variance component estimation. (DRAFT). Lincoln: Department of Agriculture/ Agricultural Research Service, 1995. 86p.

WRIGHT, D. R. et al. Comparing traditional and Bayesian analyses of selection experiments in animal breeding. Journal of Agricultural Biological and Environmental Statistics, Alexandria, v.5, n.2, p.240-256, 2000. 